\title{
La relación entre el proceso de autorregulación y el proceso de coaching*.
}

\section{The Relationship Between Self-Regulation and Coaching Process}

Recibido: 31 de agosto de 2014 | Aceptado: 21 de septiembre de 2015

\author{
JHON AleXander BaQuero BARATO** \\ MARÍA RODRÍGUEZ-MONEO *** \\ Universidad Autónoma de Madrid, España
}

doi:10.11144/Javeriana.upsy15-1.rpap

Para citar este artículo: Baquero, J., \& RodríguezMoneo, M. (2016). La relación entre el proceso de autorregulación y el proceso de coaching. Universitas Psychologica, 15(1), 141-152. http://dx.doi. org/10.11144/Javeriana.upsy15-1.rpap

* Artículo de reflexión. Este trabajo se ha desarrollado en el marco del proyecto EDU2012-37382, financiado por el Ministerio de Economía y Competitividad de España.

** Estudiante Doctoral, Facultad de Psicología. Correo electrónico: alexander.baquero@gmail.com

*** Departamento de Psicología Básica. Facultad de Psicología. Correo electrónico: maria.rodriguez@ uam.es

\section{RESUMEN}

El coaching ha tenido un importante crecimiento en los últimos años como método que facilita el logro de metas y propicia el desarrollo de habilidades. Sin embargo, existen críticas dirigidas, por un lado, a la débil fundamentación teórica que sustenta su proceso y, por otro, a la baja producción de investigaciones empíricas que expliquen los resultados de éxito y que muestren con rigor los cambios cognitivos, motivacionales y afectivos logrados por las personas que participan en este tipo de procesos. Este artículo presta especial atención al análisis de la relación de los procesos de coaching y de autorregulación. El objetivo principal se centra en plantear este vínculo como una posible solución a la necesidad de profesionalizar la práctica del coaching, de emplear técnicas basadas en la evidencia y que procedan de las investigaciones sobre los procesos psicológicos, pero, sobre todo, de encontrar un cuerpo teórico sólido que de cuenta de su éxito. También se proponen algunas posibilidades de cómo el desarrollo de la autorregulación puede verse beneficiado por las prácticas del coaching. Se plantean, por último, algunas ideas sobre la dirección de futuras investigaciones en el tema. Palabras clave

coaching; autorregulación; efectividad del coaching; evaluación del coaching; aprendizaje autorregulado

\section{A B S T R A C T}

In recent years coaching has grown significantly as a process that facilitates the achievement of goals and fosters the development of skills. However, even today it receives multiple critics regarding the lack of theoretical support, the little empirical research that supports its successful results and that shows what changes - cognitive, motivational and affective - are experienced by people who take part in this type of process. This article explores the relationship between the processes of coaching and self - regulation. Its main focus is on presenting this relationship as a possible solution to the need of professionalizing the practice of coaching, using evidence-based techniques from the research on psychological processes, but above all, finding a solid theoretical foundation that supports its success. Some ideas of how the development of self - regulation can be benefited from coaching practices are also proposed. Finally, it presents some thoughts on what could be the direction of future research on the subject.

Keywords

coaching; self-regulation; coaching effectiveness, coaching evaluation, self-regulated learning 


\section{Introducción}

En las últimas décadas se ha producido un considerable crecimiento del uso del coaching, especialmente en el campo empresarial en donde, por ejemplo, es utilizado por más del 79\% de empresas en el Reino Unido, y es considerado como una de las opciones top-five para el desarrollo del liderazgo (Lawton-Smith, 2007; Underhill, 2005). El objetivo de esta práctica está relacionado con la identificación y uso de estrategias y recursos para el logro de metas, el aprendizaje autodirigido, la adquisición de información, el desarrollo de competencias y el autoconocimiento (Baquero, 2010; Stober \& Grant, 2006). También se ha relacionado con el cambio de patrones de comportamiento asociados al cumplimiento de una meta (Bennett \& Wayne, 2009; Dai \& De Meuse, 2007), y con la transferencia de conocimientos en contextos de entrenamiento y aprendizaje (Lawton-Smith, 2007; Spencer, 2011; Vidal-Salazar, Cordón-Pozo \& Ferrón-Vílchez, 2011).

De acuerdo con Grant y Cavanagh (2004), la industria del coaching ha alcanzado un nivel de madurez importante que se refleja en tres aspectos principales: la experiencia acumulada, el número de profesionales que ingresan a este campo (Grant \& Zackon, 2004) y, por último, la sofisticación del manejo de los profesionales de recursos humanos. Además, estos autores destacan el aumento de investigaciones y tesis doctorales publicadas en revistas indexadas durante la última década, aunque señalan que la mayoría tuvieron muestras no controladas o fueron estudios de casos cuyos resultados no son generalizables.

Algunas de las investigaciones disponibles tratan de la relación entre resiliencia, coaching y habilidades de afrontamiento del estrés (Taylor, 1997), sobre el rol de la expresión de emociones positivas en coaching ejecutivo (Wachholz, 2000), sobre la estabilidad de las características de personalidad y la efectividad de una intervención específica de coaching ejecutivo (Norlander, Bergman, $\&$ Archer, 2002). Otras investigaciones (Grant \& Zackon, 2004) dan cuenta del desarrollo del perfil de un coach profesional o de la tendencia de la profesionalización de esta práctica, así como de las percepciones de los clientes (conocidos como coachees) acerca del proceso de coaching y de las cualidades y experiencia previa que ellos valoran en un coach (Fanasheh, 2003; Wasylyshyn, 2003).

Aunque la investigación en coaching ha aumentado, sin embargo son pocos los intentos por rescatar, reconocer y evaluar empíricamente la psicología del coaching (Grant, 2001). Algunos ejemplos son el estudio de Johnston (2005) que muestra la posible integración de la teoría de definición de metas con el coaching, o el estudio que realizaron Scoular y Linley (2006) que, además de la teoría de definición de metas, incluyó la relación del coaching con los tipos de personalidad.

Pese al considerable uso y reconocimiento del coaching, no obstante son múltiples las críticas que ponen de manifiesto, por un lado, la débil fundamentación teórica que sustenta esta práctica (Baquero, 2010; Brotman, Liberi, \& Wasylyshyn, 1998) y, por otro, la ausencia de suficiente soporte basado en la evidencia que permita identificar qué aspectos influyen en el logro de metas y en el desarrollo de habilidades.

Los cambios generados en los procesos psicológicos de los coachees que ocurren como resultado de una intervención de coaching, tampoco han recibido suficiente atención. Ello demuestra que ha habido menor interés en el proceso de coaching en sí mismo, es decir en lo que ocurre durante la relación entre el coach y su coachee (cliente) y que contribuye al logro de sus metas (Day, De Hann, $\&$ Sills, 2008).

En la descripción del objetivo del coaching que hacen Douglas y McCauley (1999) se resalta la importancia de generar cambios cognitivos, emocionales y comportamentales sostenibles que faciliten el logro de metas y la mejora del desempeño tanto en la vida personal como en la profesional. Así pues, resulta necesario investigar sobre los procesos cognitivos, afectivos y comportamentales que tienen lugar en las personas que reciben un proceso de coaching. En esta línea, en el año 2001 Grant mencionó que las investigaciones deberían enfocarse en cómo las intervenciones de coaching impactan en, y son mediadas por, factores socio-cognitivos tales 
como la disposición, la auto-conciencia, el insight y la autorregulación.

Más de una década después desde que Grant hizo esta afirmación, aún no conocemos suficientemente los procesos psicológicos que subyacen al proceso de coaching. Dando respuesta a esta cuestión, el propósito de este artículo es explorar teóricamente la posible vinculación entre la práctica de coaching y el proceso de autorregulación para establecer un marco de referencia que permita entender los cambios cognitivos, afectivos y motivacionales que ocurren en el coachee, y también para buscar alternativas prácticas que fomenten el desarrollo de la autorregulación en las personas.

\section{El proceso de coaching}

La palabra coaching proviene del húngaro koach que se asocia con transportar cómodamente a una persona desde donde está hasta donde quiere estar (Van Kessel, 2006, p. 393). En la actualidad no existe una definición única para coaching. Las definiciones disponibles varían considerablemente en el grado de claridad, así como en el énfasis que se hace sobre la instrucción directa o sobre la facilitación del aprendizaje autodirigido. Están además expuestas a constantes debates debido a la ausencia de datos empíricos que las sustenten (Grant, 2001, 2007).

El coaching puede entenderse como un proceso comunicativo que implica el uso de estrategias cognitivas y comportamentales para favorecer el desarrollo del potencial del coachee, y maximizar su desempeño en el logro de metas específicas (Gallwey, 2002). Laske (2004) menciona que el coaching utiliza tres procesos genéricos para ayudar a los clientes: el primero, foco de la atención, tiene un impacto de naturaleza cognitiva referido a qué es a lo que le prestan atención las personas; el segundo, prever resultados, es de naturaleza interpretativa y se relaciona con la forma en la que los coachees entienden e interpretan su vida personal y profesional. Por último, el tercer proceso, la definición de nuevos comportamientos y experiencias, se relaciona con las conductas nuevas que se definen a partir del nivel de desarrollo del coachee y/o su posición frente al logro de una meta. De acuerdo con esta definición, el rol del coach es facilitar el proceso de desarrollo del coachee de acuerdo con su presente cognitivo y emocional.

Otras definiciones de coaching incluyen características como la ausencia de enfermedad mental, la orientación hacia el logro de metas, la ejecución de acciones y el fomento de espacios de autoreflexión para la identificación de recursos que permitan alcanzar objetivos laborales o personales.

A pesar del uso masivo que tiene esta práctica en la actualidad, su profesionalidad está aún en tela de juicio (Grant, 2007). Dentro de sus limitaciones, como se ha señalado, destacan la ausencia de un cuerpo teórico sólido y de una práctica basada en evidencias, a lo que debe añadirse la baja oferta de programas de entrenamiento estructurados y regulados de acuerdo a estándares mínimos comúnmente aceptados (Baquero, 2010; Fillery-Travis \& Lane, 2007). Grant y Cavanagh (2004) afirman que mientras las instituciones de coaching han desarrollado sus propios sistemas de acreditación y códigos de ética, a menudo éstos no son aplicados apropiadamente por parte de las personas que lo ejercen.

\section{El papel de la psicología en el proceso de coaching}

Allison y Whybrow (2007), en el capítulo introductorio del handbook de coaching psicológico, mencionan que las raíces del coaching se remontan principalmente al movimiento humanista, cuyo auge se produjo en torno a los años 60. Sin embargo, estos autores indican también que el estudio de la relación entre el coaching y la psicología debe partir de los desarrollos teóricos y prácticos que se originaron a partir de 1920, con los trabajos realizados en la psicología del deporte y el acompañamiento de atletas de alto rendimiento por parte de psicólogos. De acuerdo con O'Broin y Palmer (2006), la relación existente entre la psicología del deporte y el coaching se debe abordar desde al menos tres áreas: la psicología del rendimiento, la psicología positiva y el tipo de relación que se establece entre el coach y los deportistas. 
Son múltiples las relaciones que se han planteado entre la psicología y el coaching. Por ejemplo, se ha relacionado con el conductismo (Passmore, 2007), con la terapia cognitivo-conductual (Palmer \& Szymansky, 2007), con la psicología de la Gestalt (Allison \& Whybrow, 2007), con la psicología de la motivación y la cognición (Grant, 2001), con la psicología de grupos (Thornton, 2010) y con la psicología positiva (Linley \& Harrington, 2007). Palmer y Whybrow (2006) han identificado el alto uso de técnicas cognitivas y conductuales dentro de la práctica actual del coaching.

No obstante, pese a las múltiples conexiones que se han planteado, no ha habido una rigurosa vinculación entre el coaching y los procesos psicológicos subyacentes - cognitivos, motivacionales y afectivos-, que intervienen en el logro de las metas y en el proceso del aprendizaje autorregulado.

Uno de los estudios reportados, el programa de Coach yourself (Grant \& Greene, 2001), hace una adaptación del Transtheoretical Model of Change (Prochaska \& DiClemente, 1982) y de otras aproximaciones de la psicología cognitivo-conductual, de la terapia breve enfocada en la solución y de algunos modelos de aprendizaje autorregulado, para estructurar una intervención de coaching para veinte estudiantes de posgrado de psicología durante 13 semanas.

Una vez terminada esta intervención, los autores implementaron diversos instrumentos para medir la consecución de objetivos, la depresión, la ansiedad y el estrés (Lovibond \& Lovibond, 1995), la calidad de vida (Frisch, 1994), el insight y la auto-reflexión (Grant, 2001). Los resultados muestran cómo el coaching facilita la consecución de objetivos, mejora la calidad de vida y la salud mental, así como eleva el nivel de insight y autorreflexión. Algunas limitaciones de este estudio se refieren a la ausencia de asignación aleatoria de los participantes, y el control de otras variables que posiblemente influyeron en los resultados.

Aunque la posible vinculación que existe entre el coaching y la psicología parece extensa y profunda, abarcando desde planteamientos humanistas, conductistas, cognitivos y conductuales, hasta aplicaciones prácticas como los de la psicología del deporte, psicología del trabajo, psicología de la salud, etcétera, sin embargo, debe profundizarse en el estudio de la relación coaching-psicología. Para ello, es necesario poner énfasis en la importancia de los planteamientos teóricos y prácticos que se han desarrollado en el ámbito de la psicología sobre los procesos psicológicos básicos como la motivación, la emoción, la atención, la memoria y el aprendizaje, teniendo muestras representativas, diseños de estudios experimentales y control de otras variables que pueden influir el proceso de coaching.

Grant (2001) ha establecido el marco básico de la conexión entre el coaching y la autorregulación como proceso motivacional; sin embargo, se requieren estudios empíricos de mayor profundidad para comprender esta relación y evaluar su importancia dentro de la efectividad del proceso de coaching. De acuerdo con Grant, es a través del proceso de autorregulación que se establece un vínculo entre la psicología y el coaching, ya que "el proceso de coaching trata esencialmente de ayudar a las personas a regular y dirigir sus recursos interpersonales e intrapersonales en la consecución de metas" (p.52).

De igual manera, Grant $(2001,2011)$ reconoce la importancia de desarrollar estrategias y acciones que permitan la evaluación del proceso de coaching, con el fin de satisfacer dos necesidades actuales: por un lado, demostrar su eficacia como método y, por otro, explorar el proceso, examinando cuáles son las variables intervinientes y analizando cómo influyen en el proceso. El análisis del coaching desde la psicología cognitiva y, especialmente, desde la perspectiva del proceso de autorregulación, podrían proporcionar aportaciones importantes en este sentido.

\section{El proceso de autorregulación}

Aunque la autorregulación es un proceso complejo de definir (Boekaters, Pintrich \& Zeidner, 2000), existen diferentes modelos y aproximaciones que la explican. Uno de ellos, el modelo de Zimmerman (2000) retoma la propuesta de Bandura (1986), y propone que la autorregulación se refiere a la autogeneración de pensamientos, sentimientos y acciones que son planificadas y adaptadas para 
la consecución de metas personales. Ocurre en la interacción de tres ámbitos (personal, comportamental y ambiental) y se estructura en tres fases cíclicas: planificación, desempeño y reflexión. Pintrich (2000) introduce una cuarta fase de monitorización.

La fase de planificación incluye el análisis de las tareas, el planteamiento de retos, y el establecimiento de planes de acción para el logro de las metas. Zimmerman y Schunk (2004) mencionan para esta etapa la importancia de plantear metas específicas, próximas y que constituyan un reto relacionado con el proceso de aprendizaje más que con los resultados a obtener. Asimismo, se deben considerar otros elementos motivacionales como los intereses y valores, el tipo de aproximación a las metas, las expectativas de resultado y la percepción de autoeficacia.

Por otra parte, la fase de desempeño implica dos procesos principales: el autocontrol y la autoobservación. Se caracteriza por el uso de métodos y estrategias que permitan guiar y monitorear la atención y los comportamientos, así como controlar las emociones y sentimientos, para el cumplimiento de las metas propuestas en la fase anterior.

Por último la fase de reflexión incluye la autoevaluación del proceso y de los resultados obtenidos, así como la atribución de los fallos y aciertos a factores personales y controlables que favorecen la estabilidad del nivel de motivación e interés para ejecutar comportamientos que faciliten el cumplimiento de las metas propuestas.

Otras aproximaciones (e.g., Dresel \& Haugwitz, 2005) incluyen variables como la personalidad, la motivación, el autoconcepto, el género, el conocimiento sobre estrategias y habilidades metacognitivas, que parecen influir significativamente sobre el proceso de regulación. Kuhl (1985, 1992, 2008) resalta la alta influencia que las creencias de autoeficacia y autoestima, las expectativas, los valores y otros componentes cognitivos ejercen sobre este proceso, así como la importancia de considerar las diferencias individuales en aspectos como los motivos, las necesidades y los afectos.

De acuerdo con Boekaerts y Corno (2005) el estudio del proceso de autorregulación requiere de la integración de múltiples campos de investigación psicológica, incluyendo ámbitos tales como la resolución de problemas y la toma de decisiones. De igual manera, han de tenerse en cuenta los aspectos que se describen en la psicología de la motivación, tales como patrones motivacionales, el autoconcepto, la importancia de la tarea, el nivel de dificultad de la misma y las creencias de control. Todos estos componentes influyen en el uso de las estrategias de autorregulación.

La educación, y más específicamente los procesos de aprendizaje, son los ámbitos en donde más se ha estudiado la autorregulación. De acuerdo con Pintrich y García (1994), las estrategias de autorregulación utilizadas durante el proceso de aprendizaje se clasifican en tres grupos. El primero está compuesto por las estrategias cognitivas de aprendizaje que son utilizadas para regular la adquisición de conocimientos, tales como la vinculación del nuevo conocimiento con conocimiento previo o el uso de estrategias memorísticas.

El segundo grupo se refiere al uso de estrategias metacognitivas de control entre las que se encuentran la planeación y el monitoreo de las actividades relacionadas con el aprendizaje, la evaluación de los resultados obtenidos y de los requerimientos de la tarea o de la situación. Por último, el tercer grupo hace referencia al manejo de recursos, de estrategias de control de las condiciones asociadas con el aprendizaje, tales como el tiempo y el entorno en el que tiene lugar. Como recurso adicional para el entendimiento del componente metacognitivo, la investigación ha demostrado que el mero conocimiento de las habilidades metacognitivas no garantiza que ocurra el proceso de autorregulación (Weinstein \& Hume, 1998).

Los teóricos e investigadores de la autorregulación en la educación están de acuerdo con la premisa de que los estudiantes tienen la capacidad de dirigir su proceso de aprendizaje, establecer metas y planes de acción (Boekaerts \& Corno, 2005), e indican la utilidad de la promoción del autoconcepto como una de las alternativas más eficaces para el fomento de la capacidad autorreguladora (Dresel $\&$ Haugwitz, 2005). 
En la actualidad, la importancia de la autorregulación dentro del contexto educativo se pone de manifiesto en los postulados del aprendizaje autorregulado y su relación con la competencia de aprender a aprender, que ha recibido especial atención por ser una competencia transversal en los sistemas educativos, presente desde la educación primaria hasta la universidad (Rodríguez-Moneo, 2011).

\section{La relación entre los procesos de autorregulación y coaching}

Dado que, por un lado, el coaching está teniendo un creciente uso en contextos de educación primaria y secundaria para el desarrollo de habilidades de los múltiples actores que participan en este entorno específico tales como profesores, directivos, estudiantes y padres de familia (Bamford, Mackew, \& Golawski, 2012; Knight, 2012; van Nieuwerburgh, 2012) y, por otro lado, la autorregulación ha sido ampliamente estudiada en entornos educativos, existe un contexto oportuno para establecer una posible relación entre ambos procesos.

Contrario a lo que se cree, la autorregulación no es una forma de aprendizaje, monitoreo y control exclusivamente individual; recientes trabajos en autorregulación elevan la importancia de reconocer y estudiar la influencia que tienen las interacciones sociales, tales como la búsqueda de ayuda y soporte de pares, coaches y hasta profesores (Hadwin, Miller, \& Järvelä, 2011; Zimmerman \& Schunk, 2011). De acuerdo con lo anterior, la interacción que ocurre entre una persona y un coach puede tener un impacto en la definición y el uso de estrategias de regulación que podrían ubicarse dentro de la categoría de corregulación estudiada por Hadwin, Miller, y Järvelä (2011) y que hace referencia a la mediación temporal, directa o indirecta, que tienen las interacciones sociales en el desarrollo e interiorización de prácticas de regulación.

Dentro de las fases del proceso de autorregulación propuestas por Zimmerman (2000), Pintrich (2000; 2004) y Rodríguez-Moneo (2009) es posible encontrar procesos subyacentes relacionados con el establecimiento de metas, con la planificación de la acción y la acción misma, con la monitorización y con la evaluación, principalmente. Grant (2001) ofrece una descripción básica de cómo el coaching influye en cada uno de estos procesos, resaltando la necesidad de realizar estudios empíricos que contribuyan a clarificar cómo ocurre esta influencia y cómo puede gestionarse para incrementar su efectividad. Así pues, la explicación de esta relación contribuiría a enriquecer la práctica del coaching y, al mismo tiempo, permitiría identificar métodos para fomentar el proceso de autorregulación en las personas, así como para modelar estrategias de regulación apropiadas, proveer feedback y oportunidades de práctica, entre otras cosas que son importantes en el proceso de regulación (Wolters, Benzon, \& Arroyo-Giner, 2011).

Dentro de las investigaciones realizadas en el campo de la autorregulación es posible identificar la importancia de la definición de metas y el tipo de metas que son establecidas. De acuerdo con Grant (2001), la autorregulación dirigida a una meta (goal-directed self-regulation) se caracteriza por una serie de procesos en los que un individuo define una meta, desarrolla un plan de acción, actúa, monitorea, evalúa su desempeño, y adecúa sus acciones de acuerdo con los resultados de esta evaluación para el logro de la meta. El rol del coach es facilitar la transición y el movimiento del coachee a través de todos los pasos del ciclo de la autorregulación, teniendo en cuenta que estos pasos no son independientes sino que tienen un alto grado de interrelación.

Sin embargo, pese a las evidencias que vinculan la definición de metas personales con el proceso de autorregulación (Brunstein, 1993; Omodei \& Wearing, 1990; Spence \& Grant, 2007), son insuficientes las investigaciones que han evaluado esta relación en contextos reales. Tal como menciona Kulh (2008), incluso si se estudia detalladamente el proceso cognitivo de la representación de una meta, aún falta información que dé cuenta del porqué una persona establece ciertas metas.

Adicionalmente, si bien la definición de una meta parece ser uno de los pasos más importantes en el proceso de coaching, sin embargo, hasta el momento, no existe información empírica, e in- 
cluso teórica, del tipo de metas que se plantean en el coaching y de si este último es un proceso oportuno para cualquier tipo de metas. El tipo de metas que se plantean, se corresponde con el tipo de autorregulación de la que se trate. Por ejemplo, en la autorregulación tipo top-down ${ }^{1}$, las metas están relacionadas con la motivación producto de la maestría o el dominio en un tema, los intereses personales, los valores y la satisfacción. Por otro lado, en la autorregulación tipo bottom-up $p^{2}$, las metas se relacionan con la motivación proveniente de fuentes externas o la recuperación de estados de bienestar perdidos.

Aunque la investigación sobre las metas es extensa, profundizar en este tipo de inquietudes sobre la naturaleza y tipo de metas, los factores influyentes en el establecimiento de unas metas u otras, así como el efecto de las mismas tanto en el proceso de autorregulación como en la práctica del coaching, podría proveer de información relevante para analizar con más rigor el proceso personal que cada individuo completa con una intervención de coaching.

La relación autorregulación-coaching no solo nos permite abordar la meta, sino tener en cuenta el efecto de otros componentes como los planes de acción, la aplicación y monitorización de los mismos, la evaluación, etcétera. Todos ellos son elementos comunes a ambos procesos y, sin embargo, no se ha tratado suficientemente su vinculación.

Para examinar la relación entre el proceso de autorregulación y el proceso de coaching, Grant (2001) propone un modelo sobre qué es lo regulado dentro del contexto del logro de metas, afirmando que las emociones, pensamientos, acciones y el entorno, son los cuatro factores que influyen en el alcance de metas. Estos factores deben ser incluidos

1 Este tipo de autorregulación de tipo top-down se corresponde con metas que están conectadas a metas de orden superior, que tienden a ser más valoradas. Implican programas de acción que han sido consciente e intencionalmente elegidos (Boekaerts \& Niemivirta, 2000).

2 El movimiento bottom-up se refiere a procesos de metas que reflejan la motivación el individuo para hacer algo porque las condiciones del ambiente son las adecuadas, o cuando las personas dejan de hacer algo porque las condiciones ambientales son vistas como impedimento (Boekaerts \& Niemivirta, 2000). en la exploración de la relación autorregulacióncoaching, con el fin de indagar su influencia en el logro de metas y en la efectividad del coaching en diferentes campos.

\section{Futuras direcciones en la investigación sobre coaching y autorregulación}

El uso del coaching ha aumentado considerablemente en las últimas décadas. Dentro de sus ventajas se resalta la facilitación del logro de metas y la mejora en el desempeño, tanto laboral como personal, mediante la promoción de cambios cognitivos, emocionales y del comportamiento (Douglas \& McCauley, 1999).

Para afianzar su profesionalidad, se requieren estudios empíricos para delimitar las teorías que lo fundamentan, analizar aquello que ocurre en el proceso, y mostrar su eficacia en otros ámbitos diferentes a la empresa (Kilburg, 1996; Brotman, Liberi, \& Wasylyshyn, 1998; Baquero, 2010). Explorar la vinculación que existe entre los procesos de autorregulación y coaching puede brindar un marco de referencia que, por un lado, contribuya a mejorar la práctica del coaching y, por otro, permita delimitar y definir mejor el impacto de las intervenciones de coaching en los procesos de autorregulación asociados con el logro de metas, el aprendizaje y el descubrimiento personal, que son de aplicación en múltiples contextos como la educación y las empresas.

Una posible dirección en el estudio de la relación entre coaching y autorregulación podría ocurrir en espacios académicos, enfocada en el aprendizaje autorregulado, sus componentes y modelos. Zimmerman, Kitsantas y Campillo (2005) mencionan que, desde la perspectiva social-cognitiva, "la autorregulación es un proceso auto directivo mediante el cual, los estudiantes transforman sus habilidades mentales en actividades y destrezas necesarias para funcionar en diversas áreas" (p. 2); complementan, además, que existen tres características básicas del aprendizaje autorregulado: la iniciativa personal, la perseverancia y la habilidad para adaptarse. Determinar si el coaching facilita el desarrollo de estas tres características, podría ampliar el entendimien- 
to y uso del coaching para fomentar el aprendizaje autorregulado, especialmente en estudiantes de educación superior que están expuestos a situaciones de aprendizaje más demandantes (Panadero, Alonso-Tapia, \& Huertas, 2014; Pintrich, 2004).

El estudio de la relación autorregulación-coaching también contribuiría a dotar de mayor importancia y rigor al proceso de coaching, proporcionando un impacto positivo en la fundamentación de las metodologías y prácticas que facilitan el desarrollo de la autorregulación como una capacidad básica de control en las personas, sus estados y procesos internos. Aproximaciones al aprendizaje autorregulado como la de Paris y Paris (2001), que hacen referencia a la autonomía y el control que hacen los individuos para monitorear, dirigir y regular sus acciones hacia metas de adquisición de información, desarrollo de experticia y automejora, pueden aportar información valiosa para entender cuáles son los cambios que ocurren en los procesos cognitivos de una persona como consecuencia de su participación en una intervención tipo coaching. Estos resultados pueden tener mucha importancia en múltiples contextos como la salud y la educación, y coinciden con la propuesta de Vohs y Baumeister (2004) de desarrollar investigación en autorregulación que se asocie con fundamentos teóricos y prácticos utilizables en la realidad cotidiana de las personas.

Para concluir, el uso de herramientas de evaluación procedentes de los estudios sobre la autorregulación (Wolters, Benzon \& Arroyo-Guiner, 2011) en la investigación en coaching, podría brindar una estructura sólida y de mayor rigor que permita obtener conclusiones que fortalezcan su práctica y profesionalización. De esta manera se podría contribuir a la disminución de la crítica que ha recibido el coaching referente a que, aunque el cuerpo de conocimiento disponible está en permanente crecimiento y fortalecimiento, sin embargo, la mayoría de las investigaciones empíricas disponibles emplean diseños no experimentales, en los que los grupos de participantes no tienen ningún control o se refieren a casos de estudio específicos (Grant, 2007; Palmer \& Whybrow, 2006). Asimismo, un mayor rigor en los trabajos de coaching podría con- tribuir a un mayor conocimiento sobre el proceso de autorregulación de las personas.

\section{Referencias}

Allison, J., \& Whybrow, A. (2007). Gestalt Coaching. In S. Palmer, \& A. Whybrow (Eds.), Handbook of Coaching Psychology: a guide for practitioners, New York, NY: Routledge.

Bamford, A., Mackew, N., \& Golawski, A. (2012). Coaching for parents: empowering parents to create positive relationships with their children. En C. van Nieuwerburgh (Ed.), Coaching in Education: getting better results for students, educators and parents (p. 3-24). London: Karnac Books Ltd.

Bandura, A. (1982). Self-efficacy mechanism in human agency. American Psychologist, 37(2), 122-147.

Bandura, A. (1986). Social foundations of thought and action: A social cognitive theory. Englewood Cliffs, NJ: Prentice Hall.

Baquero, J. (2010). Comparison between the training process in Coaching and the formative experience in Psychotherapy. V Jornadas Internacionales de Coaching y Mentoring: Universidad y Empresa, Universidad Politécnica de Madrid, 49-65.

Bennett, J., \& Wayne, M. (2009). Coaching in organizations: current trends and future opportunities. $O D$ Practitioner, 41(1), 2-7.

Boekaerts, M., \& Corno, L. (2005). Self-regulation in the classroom: A perspective on assessment and intervention. Applied Psychology: an international review, 54(2), 199-231.

Boekaerts, M. \& Niemivirta, M. (2000). Self-regulated learning: finding a balance between learning goals and ego-protective goals. En M. Boekaerts, P. Pintrich, \& M. Zeidner (Eds). Handbook of selfregulation, San Diego, CA: Academic Press.

Boekaerts, M., Pintrich, P., \& Zeidner, M. (Eds.) (2000). Handbook of self-regulation, San Diego, CA: Academic Press.

Brotman, L., Liberi, W., \& Wasylyshyn, K. (1998). Executive coaching: the need for standards of competence. Consulting Psychology Journal: Practice and research, 50(1), 40-46. 
Brunstein, J. (1993). Personal goals and subjective wellbeing: a longitudinal study. Journal of Personality and Social Psychology, 65, 1061-1070.

Day, A., De Hann, E., \& Sills, C. (2008). Coaches' experience of critical moments in the coaching. International Coaching Psychology Review, 3(3), 207-218.

Douglas, C., \& McCauley, C. (1999). Formal developmental relationships: A survey of organizational practices. Human Resource Development Quarterly, 10(3), 203-220.

Dresel, M., \& Haugwitz, M. (2005). The relationship between cognitive abilities and self-regulated learning: evidence for interactions with academic self-concept and gender. High ability studies, 16(2), 201-218.

Fanasheh, H. (2003). The perception of executive coaching among CEOs of America's top 500 companies. Dissertation Abstracts International, 64(03), 736.

Fillery-Travis, A., \& Lane, D. (2007). Research: does coaching work? En S. Palmer, \& A. Whybrow (Eds.), Handbook of Coaching Psychology: a guide for practitioners, New York, NY: Routledge.

Gallwey, W.T. (2002). The inner game of work: Overcoming mental obstacles for maximum performance, New York, NY: Texere.

Grant, A. (2001). Towards a psychology of coaching: the impact of coaching on metacognition, mental health and goal attainment. $\mathrm{PhD}$ dissertation at Department of Psychology, Macquarie University.

Grant, A. (2007). Past, present and future: the evolution of professional coaching and coaching psychology. In S. Palmer, \& A. Whybrow (Eds.), Handbook of Coaching Psychology: a guide for practitioners, New York, NY: Routledge.

Grant, A. (2011). Developing an agenda for teaching coaching psychology. International Coaching Psychology Review, 6(1), 84-99.

Grant, A., \& Cavanagh, M. (2004). Toward a profession of coaching: sixty-five years of progress and challenges for the future. International Journal of Evidence Based Coaching and Mentoring, 2(1), 1-16.

Grant, A., \& Greene, J. (2001). Coach yourself: Make real change in your life. London: Momentum Press

Grant, A., \& Zackon, M. (2004). Executive, workplace and life coaching: findings from a large-scale survey of international coach federation members.
International Journal of Evidence Based Coaching and Mentoring, 2(2), 1-15.

Hadwin, A. F., Miller, M., \& Järvelä, S. (2011). Selfregulated, co-regulated, and socially shared regulation of learning. In B. Zimmerman \& D. Schunk (Eds.), Handbook of self-regulation of learning and performance, New York, NY: Routledge.

Johnston, S. (2005). Applying goal setting theory to coaching. The coaching psychologist, 1(2), 10-12.

Kilburg, R. (1996). Toward a conceptual understanding and definition of executive coaching. Consulting Psychology Journal: Practice and research, 48(2), 134-144.

Knight, J. (2012). Coaching to improve teaching: using the instructional coaching model. En C. van Nieuwerburgh (Ed.), Coaching in Education: getting better results for students, educators and parents ( $\mathrm{p}$. 3-24). London: Karnac Books Ltd.

Kuhl, J. (1985). Volitional mediators of cognition-behavior consistency: Self-regulatory processes and action versus state orientation. En J. Kuhl \& J. Beckmann (Eds.), Action control: From cognition to behavior, Heildelberg: Springer.

Kuhl, J. (1992). A theory of self-regulation: Action versus state orientation, self-discrimination, and some applications. Applied Psychology: An International Review, 41, 97-129.

Kuhl, J. (2008). Individual differences in self-regulation. En J. Heckhausen, \& H. Heckhausen (Eds), Motivation and action, New York, NY: Cambridge

Laske, O. (2004). Can evidence-based coaching increase ROI? International Journal of Evidence Based Coaching and Mentoring, 2(2), 41-53.

Lawton-Smith, C. (2007). Coaching: is it just a new name for training? International Journal of Evidence Based Coaching and Mentoring, special issue, summer, 1-18.

Linley, P., \& Harrington, S. (2007). Integrating positive psychology and coaching psychology: shared assumptions and aspirations? En S. Palmer, \& A. Whybrow (Eds.), Handbook of Coaching Psychology: a guide for practitioners, New York, NY: Routledge.

Palmer, S., \& Whybrow, A. (2006). The coaching psychology movement and its development within the British Psychological Society. International Coaching Psychology Review 1(1), 5-11. 
Palmer, S., \& Szymansky, K. (2007). Cognitive Behavioural Coaching. En S. Palmer, \& A. Whybrow (Eds.), Handbook of Coaching Psychology: a guide for practitioners, New York, NY: Routledge.

Palmer, S., \& Whybrow, A. (Eds.) (2007). Handbook of coaching psychology: a guide for practitioners, New York, NY: Routledge.

Panadero, E., Alonso-Tapia, J., \& Huertas, J-A. (2014). Rubrics vs self-assessment scripts: effects on first year university students' self-regulation and performance. Infancia y Aprendizaje / Journal for the Study of Education and Development, 37(1), 149-183.

Paris, S., \& Paris, A. (2001). Classroom applications of research on self-regulated learning. Educational Psychologist, 36(2), 89 -101.

Parsloe, E. (1995). Coaching, mentoring and assessing: A practical guide for developing competence, New York, NY: Kogan.

Passmore, J. (2007). Behavioural Coaching. En S. Palmer, \& A. Whybrow (Eds.), Handbook of Coaching Psychology: a guide for practitioners, New York, NY: Routledge.

Pintrich, P. (2000). The role of goal orientation in selfregulated learning. En M. Boekaerts, P., Pintrich, \& M. Zeidner (Eds.), Handbook of self-regulation, San Diego, CA: Academic Press.

Pintrich, P. (2004). A conceptual framework for assessing motivation and self-regulated learning in college students. Educational Psychology Review, 16(4), 385-407.

Pintrich, P., \& Garcia, T. (1994). Self-regulated learning in college students: Knowledge, strategies and motivation. En P. R. Pintrich, D. R. Brown \& C. E. Weinstein (Eds.), Student motivation, cognition, and learning: Essays in honor of Wilbert J. McKeachie (pp. 113-133). Hillsdale, NJ: Lawrence Erlbaum Associates.

Norlander, T., Bergman, H., \& Archer, T. (2002). Relative constancy of personality characteristics and efficacy of a 12-month training program in facilitating coping strategies. Social Behavior $\mathcal{E}$ Personality, 30(8), 773-783.

O’Broin, A. \& Palmer, S. (2006). Win-win situation? Learning from parallels and differences between coaching psychology and sport psychology. The Coaching Psychologist, 2(3), 17-23.
Omodei, M., \& Wearing, A. (1990). Need satisfaction and involvement in personal projects: Towards an integrative model of subjective well-being. Journal of Personality and Social Psychology, 59, 762-769.

Rodríguez-Moneo, M. (2009). Motivar para aprender en situaciones académicas. En G. Romero \& A. Caballero (Eds.). La crisis de la escuela educadora, Barcelona: Laertes.

Rodríguez-Moneo, M. (2011). El proceso de enseñanza y aprendizaje de competencias. En F. Labrador \& R. Santero (Eds.), Evaluación global de los resultados del aprendizaje en las titulaciones dentro del Espacio Europeo de Educación Superior, Madrid: Dykinson.

Scoular, A., \& Linley, A. (2006). Coaching, goal setting and personality type: what matters? The Coaching Psychologist, 2(1), 9-11.

Spence, G., \& Grant, A. (2007). Professional and peer life coaching and the enhancement of goal striving and well-being: An exploratory study. The Journal of Positive Psychology, 2(3), 185-194.

Spencer, L. (2011). Coaching and training transfer: a phenomenological inquiry into combined trainingcoaching programmes. International Journal of Evidence Based Coaching and Mentoring, 5, 1-18.

Stober, D. \& Grant, A. (2006). Toward a contextual approach to coaching models. En D.R. Stober \& A.M. Grand (Eds.), Evidence-based Coaching Handbook (pp. 355-366). New York, NY: Wiley

Taylor, L. (1997). The relation between resilience, coaching, coping skills training, and perceived stress during a career-threatening milestone. DAIB 58/05, 2738.

Thornton, C. (2010). Group and Team Coaching: The Essential Guide (Essential Coaching Skills and Knowledge). New York, NY: Routledge.

Underhill, B. (2005). High impact executive coaching: executive summary. Executive Development Associates, Inc.

Van Kessel, L. (2006). Coaching, a field for professional supervisors? Ljetopis SocijalnorRada, 14(2), 387-342.

Van Nieuwerburgh, C. (2012). Coaching in education: an overview. En C. van Nieuwerburgh (Ed.), Coaching in Education: getting better results for students, educators and parents (p. 3-24). London: Karnac Books Ltd. 
Vidal-Salazar, M., Cordón-Pozo, E., \& Ferrón-Vílchez, V. (2011). Efectividad del coaching en los procesos de mejora de gestión de empresas. Universia Business Review, tercer trimestre 2011, 82-101.

Vohs, K., \& Baumeister, R. (2004). Understanding selfregulation: an introduction. En R.F. Baumeister, \& K.D. Bohs (Eds) . Handbook of self-regulation, New York, NY: Guilford.

Wachholz, P. (2000). Investigating a corporate coaching event: Focusing on collaborative reflective practice and the use of displayed emotions to enhance the supervisory coaching process. Dissertation Abstracts International, A (Humanities and Social Sciences), 61(2), 587.

Wasylyshyn, K. (2003). Executive Coaching: An outcome study. En Consulting Psychology Journal: Practice $\mathcal{E}$ research, 55(2), 94-106.

Weinstein, C., \& Hume, L. (1998). Study strategies for lifelong learning. American Psychological Association: Washington.

Wolters, C., Benzon, M., \& Arroyo-Giner, C. (2011) Assesing strategies for the Self-regulation of Mo- tivation. In B. Zimmerman \& D. Schunk (Eds.), Handbook of self-regulation of learning and performance, New York, NY: Routledge.

Zimmerman, B. (2000). Attaining self-regulation: a social cognitive perspective. En M. Boekaerts, P. Pintrich, \& M. Zeidner (Eds.), Handbook of selfregulation, San Diego, CA: Academic Press.

Zimmerman, B. \& Schunk, D. (2004). Self-regulating intellectual processes and outcomes: a social cognitive perspective. En D. Y. Dai \& R. J. Stenberg (Eds.), Motivation, emotion, and cognition. Mahwah, NJ: Lawrence Erlbaum Associates.

Zimmerman, B. \& Schunk, D. (2011). Self-regulated learning and performance: an introduction and an overview. In B. Zimmerman \& D. Schunk (Eds.), Handbook of self-regulation of learning and performance, New York, NY: Routledge.

Zimmerman, B., Kitsantas, A., \& Campillo, M. (2005). Evaluación de la autoeficacia regulatoria: una perspectiva social cognitiva. Laboratorio de evaluación psicológica y educativa. Facultad de Psicología, Universidad Nacional de Córdoba, Argentina. 
